\title{
Management of product variety in cellular manufacturing systems
}

\author{
M. Selim Akturk • H. Muge Yayla
}

Received: 21 July 2004 / Revised: 15 June 2005 / Accepted: 1 July 2005

(C) Springer Science + Business Media, LLC 2006

\begin{abstract}
In today's markets, non-uniform, customized products complicate the manufacturing processes significantly. In this paper, we propose a cellular manufacturing system design model to manage product variety by integrating with the technology selection decision. The proposed model determines the product families and machine groups while deciding the technology of each cell individually. Hedging against changing market dynamics leads us to the use of flexible machining systems and dedicated manufacturing systems at the same facility. In order to integrate the market characteristics in our model, we proposed a new cost function. Further, we modified a well known similarity measure in order to handle the operational capability of the available technology. In the paper, our hybrid technology approach is presented via a multi-objective mathematical model. A filtered-beam based local search heuristic is proposed to solve the problem efficiently. We compare the proposed approach with a dedicated technology model and showed that the improvement with the proposed hybrid technology approach is greater than $100 \%$ in unstable markets requiring high product varieties, regardless of the volumes of the products.
\end{abstract}

Keywords Cellular manufacturing systems · Technology selection · Product variety

\section{Introduction}

Business world of the $21^{\text {st }}$ century witnesses an expanding global competition with increased variety of products and low demand. Old manufacturing technologies fail to meet the increasing demand for customized production. The concept of Group Technology (GT) had risen to reduce WIP inventories, setups, material handling distances, and batch sizes. Cellular manufacturing system design (CMSD) is the application of GT at the production floor. In literature, different approaches are proposed to solve the CMSD problem. In their review paper, Selim et al. (1998) critically evaluate the research on cell formation. They highlight the need for incorporation of manufacturing flexibility as a strategic and operational competitive

M. S. Akturk $(\varangle) \cdot$ H. M. Yayla

Dept. of Industrial Engineering, Bilkent University, 06800 Bilkent, Ankara, Turkey

e-mail: akturk@bilkent.edu.tr 
weapon in cellular manufacturing systems. The general assumption of stable markets with highly standardized products with high and stable demand patterns is no more valid. Thus, we propose a new model to incorporate the market information effectively, while designing a flexible and efficient manufacturing system. We are inspired by the case study of Venkatesan (1990). The author reports the dissatisfaction of CMSD to cope with the increasing product variety and the actions taken by the factory to become more flexible while maintaining the benefits of cellular manufacturing. Ramdas (2003) provides a framework for managerial decisions about variety. The author states that an increase in long run profits depends on how the firm's functions are managed to implement variety.

On the other hand, increased product variety, customized and instable product designs, and international competition lead to the development of new manufacturing technologies. Productivity, quality, and flexibility are critical measures of manufacturing performance for justifying the investment in computer integrated manufacturing systems. Flexibility is the key concept used in the design of modern automated manufacturing systems. In literature, there are number of studies that analyze the trade off between flexible technology and dedicated technology. Gupta and Goyal (1989) provide a comprehensive review of the literature on flexibility. Singhal et al. (1987) define the benefits of flexible technologies as the ability to respond quickly to changes in design and demand, lower direct manufacturing costs, improved quality, economies of scope, and flexibility in scheduling.

Technology selection problem deals with selecting the best alternative among available technologies while designing a manufacturing system. In this paper, the proposed model makes use of the automated technologies while keeping the dedicated technologies as an alternative. There exist studies that considered technology selection problem simultaneously with the facility location and capacity acquisition problems. Rajagopalan (1993), Li and Tirupati (1994), and Verter and Dasci (2002) studied technology selection problem integrated in the facility location and capacity expansion decision models. In general, technology selection models do not deal with specific manufacturing system design issues. As far as we know, the cellular manufacturing and technology selection problems are studied separately in the literature; Bokhorst et al. (2002) is a notable exception. They present a mixed integer programming model to solve the part-operation allocation problem together with the investment appraisal of CNC machines to maximize the net present value over a specified planning horizon. Recently Abdi and Labib (2004) studied the manufacturing flexibility to sustain competitiveness via grouping the products into families. They focus on the design for new product introduction and analyze reconfigurable manufacturing systems. Their model does not include machining decisions. Wicks and Reasor (1999) studied on a multi period formulation that incorporates demand uncertainty. They suggested a model that minimizes the relocation costs of a dedicated cellular manufacturing system, focusing on the expected demand fluctuations. In our model we focus on the design problem such that the set of parts and their attributes (including part volume, location in the life cycle and number of design changes) are all known at the beginning of the problem. We introduce flexible systems together with dedicated systems in the same cellular manufacturing system. It is known that the flexible manufacturing systems are more robust to fluctuations in demand and design than dedicated systems given that they have higher processing flexibilities. Although we do not test robustness in our study, we assume that it is a side benefit of flexibility discussed here. We show the significance of hybrid technology implementation at the production floor to cope with the variations in the market at the present. On the other hand, Garbie et al. (2005) propose a strategy to introduce a new part into an existing cellular manufacturing system. They provide new similarity, productivity and flexibility measures that can be used at any time in the future to evaluate and quantify the effectiveness of the system. 
The product variety management should be effectively put into operation in today's world. Today's high product variety environments come not only with the higher number of differentiated products with higher number of design changes but also with higher variations in demand and lower volumes of production. We believe that the new era of higher product variety environments should be analyzed together with all the associated costs. The proposed model captures all these characterizations of the new manufacturing environment. The model determines the product families and machine groups while deciding the technology of each cell individually such that we can analyze product variety management in cellular manufacturing systems by integrating technology selection decision in the model. In order to hedge against the market variability we make use of flexible machining systems and dedicated manufacturing systems at the same facility. Our hybrid technology approach allows both types of manufacturing systems exist in the same facility (but in different cells). The model forces the parts with high demand and/or design variability to the cells with flexible machining systems. The parts with stable demand and/or design patterns are processed in dedicated manufacturing cells. The economies of scope advantage of flexible machining systems are integrated with the economies of scale advantage of dedicated manufacturing systems in the hybrid technology approach. Further, in order to integrate the market characteristics in our model, we propose a new cost function. In the paper, our hybrid technology approach is presented via a multi-objective mathematical model. Few studies (e.g. Akturk and Balkose (1996) and Suresh and Slomp (2001)) have formulated the cell formation problem by a multi-objective modeling approach, which is another contribution to part-machine grouping problem. A filtered-beam based local search heuristic is proposed to solve the problem efficiently.

The remainder of the paper is organized as follows. In Section 2, we define the scope of the study with underlying assumptions and state a mathematical formulation of the problem. In Section 3, we present the proposed solution procedure. The results of the experimental design to test the efficiency of the proposed algorithm are discussed in Section 4. Finally, Section 5 concludes the discussion about the study and some future research directions are provided.

\section{Problem statement}

In this study, an integrated approach is proposed to solve the cell design and technology selection problems simultaneously during the design of an advanced manufacturing system. The notation that will be used throughout the paper is as follows:

$\begin{array}{lll}N & : & \text { number of parts } \\ F M & : & \text { set of flexible machine types } \\ D M & : & \text { set of dedicated machine types } \\ O & : & \text { number of operations } \\ K & : & \text { maximum number of cells } \\ D_{i} & : & \text { annual demand of part } i \\ M C M_{m o} & : & 0-1 \text { binary parameter that equals to } 1 \text { if machine } m \text { is capable of performing } \\ & & \text { operation } o \\ P R M_{i o} & : & 0-1 \text { binary parameter that equals to } 1 \text { if part } i \text { requires operation } o \\ c_{i d} & : & \text { cost of assigning part } i \text { to a dedicated cell } \\ c_{i f} & : \text { cost of assigning part } i \text { to the FMS cell } \\ a_{v o l} & : & \text { production volume coefficient of the part } \\ a_{\sigma} & : \text { demand variation coefficient of the part } \\ a_{d e s} & : \text { design stability coefficient of the part }\end{array}$




\begin{tabular}{|c|c|}
\hline$D M J_{i j}^{d}$ & : dissimilarity of parts $i$ and $j$ in a dedicated cell \\
\hline$D M J_{i j}^{f}$ & $:$ dissimilarity of parts $i$ and $j$ in a flexible cell \\
\hline$I n v_{m}$ & $\begin{array}{l}\text { : annual investment cost of machine } m \text { (total investment costs/expected } \\
\text { lifetime) }\end{array}$ \\
\hline Maint $_{m}$ & $:$ annual maintenance cost of machine $m$ \\
\hline Labor $_{d}$ & : cost of one labor in a dedicated cell \\
\hline Labor $_{f}$ & : cost of labor operating the FMS cell \\
\hline OR & : operator ratio \\
\hline$S R$ & : supplementary labor ratio \\
\hline$U B K$ & : upper bound on the cell size \\
\hline$H$ & : a very large constant \\
\hline TCap & : theoretical capacity of machines \\
\hline Ptime $_{i m o}$ & : processing time of operation $o$ of part $i$ on machine $m$ \\
\hline Ltime $_{i m}$ & $:$ load-unload time of part $i$ on machine $m$ \\
\hline$\alpha_{m}$ & : upper utilization limit of machine $m$ \\
\hline$\beta_{m}$ & : lower utilization limit of machine $m$ \\
\hline $\operatorname{Tot} L_{d k}$ & : total cost of labor in dedicated cell $k$ \\
\hline $\operatorname{Tot} L_{f k}$ & $:$ total cost of labor in FMS cell $k$ \\
\hline$U t i l_{m k}$ & $:$ utilization of machine type $m$ in cell $k$ \\
\hline Excess $_{m k}$ & : excess capacity of machine type $m$ in cell $k$ \\
\hline Norm $f_{h}$ & : normalized value of objective $h$ \\
\hline GMinf $_{h}$ & : global minimum value of objective $h$ \\
\hline $\operatorname{LMaxf}_{h}$ & : local maximum value of objective $h$ \\
\hline
\end{tabular}

In the model, we assume there exist two available technologies: flexible and dedicated manufacturing systems. Machine Capability Matrix (MCM) is a 0-1 matrix presenting the operational capabilities of the machines. The matrix is composed of two blocks: one identity matrix representing the dedicated machines and an irregular 0-1 matrix that shows the capability of flexible machines. As the number of 1's in a flexible machine's row increases, we assume the machine gets more flexible, since the machine flexibility can be measured by the number of operations that can be handled by that machine. Part Requirement Matrix (PRM) is a 0-1 matrix representing the processing requirements of the parts. The processing time of each operation of each part on each machine (Ptime imo $_{\text {o }}$ ) is predetermined. Processing times of parts are important not only because they are utilized in determining the number of machines required of each type, but also they form a basis for the technology decision via determining the throughput times. The processing times of flexible machines are longer compared to that of dedicated machines. Load and unload times (Ltime im $_{\text {) }}$ are assumed to be equal for each part-machine pair and known a priori, but on the average, the load/unload times of a flexible machine is longer compared to that of a dedicated machine. However, each part should be loaded and unloaded on a dedicated machine for each operation while the flexible machines can handle a number of operations with a single load/unload. Since there are several machines in a dedicated cell, total load/unload time of a flexible cell will be shorter than it is in dedicated cells. We assume that one operator is enough to handle the required activities in a flexible cell whereas each machine needs an operator in a dedicated cell. The machine investment, maintenance, and labor costs are assumed to be known.

In order to integrate the market characteristics in our model, we proposed a new cost function. This is the first study to assign costs to design instabilities and demand variations. The costs associated with product variety is quantified via the differences in three characteristics of a product in a production environment: production volume, demand pattern, and design 且 Springer 
Table 1 Proposed product variety cost function parameters

\begin{tabular}{|c|c|c|c|}
\hline Production volume & $a_{\text {vol }}$ & Effect in $c_{i d}$ & Effect in $c_{i f}$ \\
\hline High & 1 & $a_{\text {vol }}$ & $4-a_{v o l}$ \\
\hline Medium & 2 & $a_{v o l}$ & $4-a_{v o l}$ \\
\hline Low & 3 & $a_{v o l}$ & $4-a_{v o l}$ \\
\hline Position in the life-cycle & $a_{\sigma}$ & Effect in $c_{i d}$ & Effect in $c_{i f}$ \\
\hline P3, P4 & 1 & $a_{\sigma}^{2}$ & $\left(4-a_{\sigma}\right)^{2}$ \\
\hline $\mathrm{P} 2, \mathrm{P} 5$ & 2 & $a_{\sigma}^{2}$ & $\left(4-a_{\sigma}\right)^{2}$ \\
\hline P1 & 3 & $a_{\sigma}^{2}$ & $\left(4-a_{\sigma}\right)^{2}$ \\
\hline$\frac{\text { Number of design changes }}{\text { Part age }}$ & $a_{\text {des }}$ & Effect in $c_{i d}$ & Effect in $c_{i f}$ \\
\hline Low & 1 & $a_{d e s}^{3}$ & $\left(4-a_{d e s}\right)^{3}$ \\
\hline Medium & 2 & $a_{\text {des }}^{3}$ & $\left(4-a_{d e s}\right)^{3}$ \\
\hline High & 3 & $a_{\text {des }}^{3}$ & $\left(4-a_{d e s}\right)^{3}$ \\
\hline
\end{tabular}

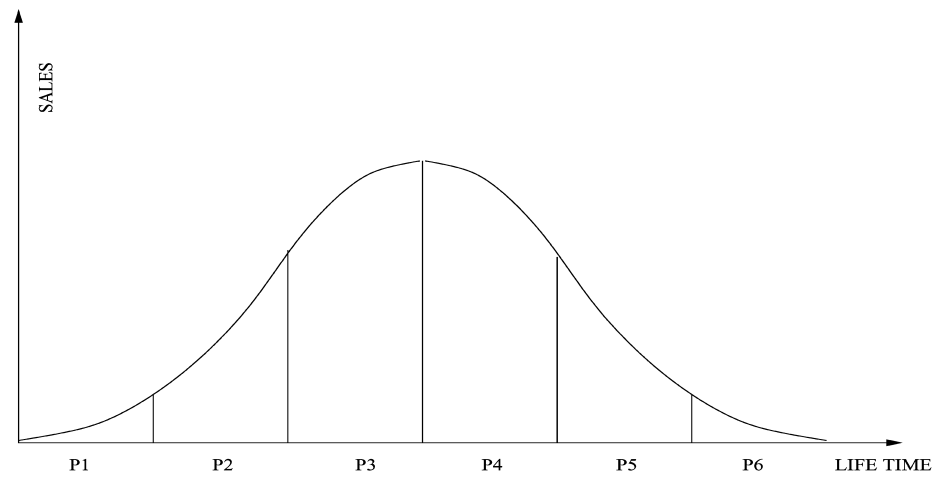

Fig. 1 Traditional product life cycle

stability. An important question is how we can quantify and also unify these intangible measures as discussed below. The proposed product variety costing scheme is given in Table 1.

Production volume, $a_{v o l}$ : A part can have a high production volume whereas another, but operationally similar, part can have a low production volume. If these two parts are assigned in the same cell, the frequent and interrupting setup requirements can become a burden contradicting that setup should have been an advantage of cellular manufacturing. Low volume parts should be directed to FMS cells, whereas the high-volume parts to the dedicated cells.

Demand pattern, $a_{\sigma}$ : In the early stages of the typical life cycle of a product (periods P1 and P2 of Fig. 1), demand variation is high. However, the demand has much less variation during the saturation phase (periods $\mathrm{P} 3$ and $\mathrm{P} 4$ ). It is not wrong to assume that there exists no product of period P6. To allocate resources for a newly introduced part may not end up with satisfactory results. We should benefit from the flexibility of flexible manufacturing cells, and force the high variation parts to be assigned to FMS cells. The importance of the variation cost is emphasized by assigning the square power of the coefficients as the cost values. The coefficients are based on the life cycle positions of the parts. 
Design stability, $a_{d e s}$ : Many parts have evolving designs to satisfy the changing demands of customers. The design is said to be stable when the processing requirements are not changed often. When it is evolving, new operations may be added or some may be discarded from the routing frequently. The flexible manufacturing systems are more robust to fluctuations in design than the dedicated systems given that they have higher processing flexibilities. There is positive probability that the flexible cell can handle the added operation without any change whereas this probability is much lower in a dedicated cell. Thus, we should assign an evolving part to a dedicated cell if and only if we have no other alternative. The operational requirements of each part are an important attribute of the CMSD problem, which is quite sensitive to the design stability. The significance and superiority of the design stability is emphasized by assigning the triple power of the coefficients as the cost values. The coefficients are based on the average number of design changes per life time unit of the product as presented in Table 1.

After identification of the values of coefficients and associated cost values of parts, final product variety cost function values are calculated for each part. Having assigned different weights to the attributes, we make use of the simplicity and power of additivity in our proposed cost function. The following $c_{i d}$ and $c_{i f}$ parameters can be interpreted as complementary costs, such that the more we prefer to assign a part to the flexible cell, the less we prefer to assign that part to a dedicated cell, and vice versa.

$$
c_{i d}=a_{v o l}+a_{\sigma}^{2}+a_{d e s}^{3} \quad \text { and } \quad c_{i f}=\left(4-a_{v o l}\right)+\left(4-a_{\sigma}\right)^{2}+\left(4-a_{d e s}\right)^{3}
$$

The cost function have several important missions to be used in the solution procedure. It is basically used as a surrogate objective function of the model. Further, it provides us a strong basis for the selection of technology for each cell. The possible values of $c_{i d}$ and $c_{i f}$ range in between [3, 39]. For example, $c_{i d}=3$ and $c_{i f}=39$ if there is a part $i$ with a high production volume $\left(a_{v o l}=1\right)$, position in the life-cycle is P3 or P4 $\left(a_{\sigma}=1\right)$ and the number of design changes is low $\left(a_{d e s}=1\right)$. Similarly, $c_{i d}=39$ and $c_{i f}=3$ if there is a part $i$ with a low production volume $\left(a_{v o l}=3\right)$, newly introduced to market $\left(a_{\sigma}=3\right)$ with high design changes $\left(a_{d e s}=3\right)$. Therefore the parts with small $c_{i d}$ values are most probable members of families being processed on dedicated cells, and the ones with high $c_{i d}$ (or with low $c_{i f}$ ) values are most probably selected for being processed on flexible cells. When we plot all the possible values of these cost functions, we observe that the $c_{i d}$ cost value of 15 is a meaningful candidate to represent a threshold value to choose between dedicated and flexible technologies. This threshold value is utilized at critical points in the algorithm.

In addition to the new cost function, we modified a well known operational similarity measure in order to handle the operational capability of available technology. Jaccard coefficient (JC) is the most often used coefficient in the similarity context. It is not only a powerful coefficient but also very simple and effective. JC calculates the similarity between two machines $m$ and $n$ as follows:

$$
J C_{m n}=\frac{\text { number of parts visiting both machines }}{\text { total number of parts processed on these machines }} \quad \text { where } 0 \leq J C_{m n} \leq 1
$$

The main assumption underlying this coefficient is that a specific operation can be handled by a specific machine, and whenever a part requires that operation, it has to visit that machine. However, with the available flexible manufacturing technology, an operation can be handled by several different types of machines. Similarity context should be adapted to the technological advancements. The proposed coefficient calculates the dissimilarity between two parts $i$ and $j$ in two stages based on $J C$ as follows: 
1. A hypothetical manufacturing cell is designed to find the minimum number of machines required to produce two parts in the same cell. Calculations are based on the average product variety costs $\left(c_{i d}\right.$ 's) of the two parts. If the average cost of parts $i$ and $j$ is lower than the threshold value, the dedicated block of the MCM is available for the calculation of the similarity and hence the dissimilarity $\left(D M J_{i j}^{d}\right)$. Otherwise, if the average cost is high, i.e., parts are more likely to meet in a flexible cell, flexible block of MCM is used to calculate the dissimilarity of parts $\left(D M J_{i j}^{f}\right)$.

2. After the hypothetical cell design, the Dissimilarity Coefficients $\left(D M J_{i j}\right)$ are calculated as follows:

$$
D M J_{i j}=1-\frac{\text { number of machines where both parts have an operation }}{\text { total number of machines in the hypothetical cell }},
$$

where $0 \leq D M J_{i j} \leq 1$

Example 1. Let the threshold value be 15 and PRM and MCM data are given as follows:

\begin{tabular}{r|ccc|c} 
PRM & op1 & op2 & op3 & Variety Costs $\left(c_{i d}\right)$ \\
\hline p1 & 1 & 1 & 1 & 15 \\
p2 & 1 & 1 & 0 & 5 \\
p3 & 0 & 1 & 0 & 33
\end{tabular}

\begin{tabular}{r|ccc}
$\mathrm{MCM}$ & op1 & op2 & op3 \\
\hline $\mathrm{dm} 1$ & 1 & 0 & 0 \\
$\mathrm{dm} 2$ & 0 & 1 & 0 \\
$\mathrm{dm} 3$ & 0 & 0 & 1 \\
$\mathrm{fm} 1$ & 1 & 0 & 1 \\
$\mathrm{fm} 2$ & 0 & 1 & 1
\end{tabular}

Average Cost $_{p 1 p 2}=\frac{15+5}{2}=10<15$. Since the average cost is less than the preselected threshold value, these two parts $p 1$ and $p 2$ can be assigned to a dedicated cell composed of machines $\mathrm{dm} 1, \mathrm{dm} 2$ and $\mathrm{dm} 3$ so that

$$
D M J_{12}^{d}=1-\frac{|d m 1+d m 2|}{|d m 1+d m 2+d m 3|}=1-\frac{2}{3}=\frac{1}{3} .
$$

On the other hand, for parts $p 1$ and $p 3$, Average Cost $_{p 1 p 3}=\frac{15+33}{2}=24>15$, and hence they can be processed by the flexible machines so that

$$
D M J_{13}^{f}=1-\frac{|f m 2|}{|f m 2+f m 1|}=1-\frac{1}{2}=\frac{1}{2} .
$$

As shown in the numerical example, we calculate a dissimilarity value for each part pair based on their variety costs and operational similarities.

Under the assumptions and definitions presented, the decision variables of the model are the following:

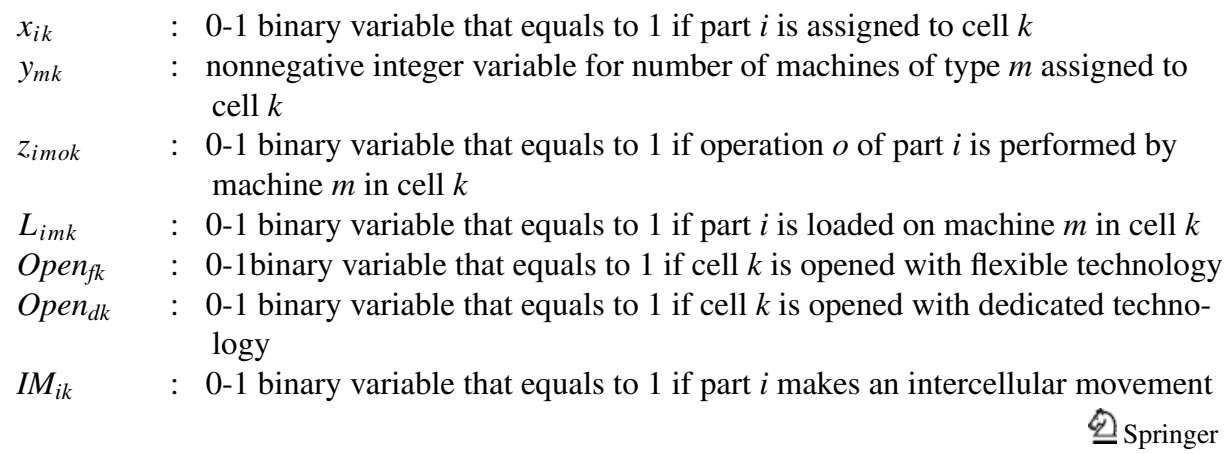


to cell $k$

The mathematical formulation of the problem is as follows:

$$
\begin{aligned}
\min f_{1}= & \sum_{i, j, f} x_{i f} \cdot x_{j f} \cdot D M J_{i j}^{f}+\sum_{i, j, d} x_{i d} \cdot x_{j d} \cdot \text { DMJ }_{i j}^{d} \\
\min f_{2}= & \sum_{i} x_{i f} \cdot c_{i f}+\sum_{i} x_{i d} \cdot c_{i d} \\
\min f_{3}= & \sum_{i, m, o, k} z_{\text {imok }} \cdot \text { Ptime }_{i m o}+\sum_{i, m, k} L_{i m k} \cdot 2 \cdot \text { Ltime }_{i m} \\
\min f_{4}= & \sum_{m, k} y_{m k} \cdot\left(\text { Inv }_{m}+\text { Maint }_{m}\right)+\sum_{k} \text { Open }_{f k} \cdot \text { Labor }_{f} \\
& +\sum_{m, k} \text { Open }_{d k} \cdot y_{m k} \cdot \text { Labor }_{d} \\
\min f_{5}= & \sum_{i, k} \text { IM }_{i k}
\end{aligned}
$$

$$
\begin{aligned}
& \text { subject to } \sum_{k=1}^{K} x_{i k}=1 \quad \forall i=1, \ldots, N \\
& y_{m k}=0 \quad \forall m \in D M \text { and flexible cell } k \\
& y_{m k}=0 \quad \forall m \in F M \text { and dedicated cell } k \\
& H \cdot \text { Open }_{f k} \geq \sum_{i} x_{i k} \geq \text { Open }_{f k} \quad \forall \text { flexible cell } k \\
& H \cdot \text { Open }_{d k} \geq \sum_{i} x_{i k} \geq \text { Open }_{d k} \quad \forall \text { dedicated cell } k \\
& z_{\text {imok }} \leq P R M_{i o} \cdot y_{m k} \cdot M_{\text {mo }} \quad \forall i, m, o, k \\
& \sum_{m, k} z_{\text {imok }}=P R M_{\text {io }} \quad \forall i, o \\
& \text { TCap } \cdot \beta_{m} \cdot y_{m k} \leq \sum_{i, o} z_{\text {imok }} \cdot \text { Ptime }_{\text {imo }} \cdot D_{i} \\
& \leq \text { TCap } \cdot \alpha_{m} \cdot y_{m k} \quad \forall m, k \\
& \sum_{m} y_{m k} \leq U B K \cdot \text { Open }_{f k} \quad \forall \text { flexible cell } k \\
& \sum_{m} y_{m k} \leq U B K \cdot \text { Open }_{d k} \quad \forall \text { dedicated cell } k \\
& H \cdot L_{i m k} \geq \sum_{o} z_{i m o k} \geq L_{i m k} \quad \forall i, m, k \\
& H \cdot I M_{i k} \geq\left(\sum_{m, o} z_{i m o k}\right) \cdot\left(1-x_{i k}\right) \geq I M_{i k} \quad \forall i, k
\end{aligned}
$$


The model is structured to be multi-objective. Our aim is to find a good compromise solution in order to satisfy all objectives. When considered alone, the minimization of objective 1 results in part families that have the best operational similarities among the member parts and objective 2 results in two groups of parts, that are processed either in flexible or dedicated cells. Each part is preferred to be placed in the group of parts in which the associated variety cost term is smaller. The objective 3 is significant because it has two contradicting parts regarding the technology selection at the same time. In the flexible cells processing times will be longer favoring dedicated cells, whereas in dedicated cells load-unload times will become a burden favoring flexible cells. In the objective 4, the critical measures for the technology selection decision are analyzed. The two contradicting parts of the function (investment and labor costs) form a strong basis for technology selection decision. In cellular manufacturing, one of the most critical objectives is the minimization of intercellular movements. The objective 5 is in conflict with other objectives in order not to allow exceptional parts.

Each part is a member of only one family in the system by the constraint 6 . The constraints 7 and 8 assure that none of the dedicated machines are assigned in the FMS cell, in order not to destroy the total computer integration in the cell, and dedicated cells are totally composed of dedicated machines. The constraints 9 and 10 control the opening of the cells, whereas 11 and 12 control the operational allocation of parts. Each necessary operation of a part should be handled by a machine in any one of the cells. To be able to assign an operation of a part to a machine in a cell $\left(z_{\text {imok }}=1\right)$, three conditions should hold: Operation should be necessary for the part $\left(P R M_{i o}=1\right)$, at least one machine should exist in that cell $\left(y_{m k} \geq 1\right)$, that machine should be capable of that operation $\left(M C M_{m o}=1\right)$. Upper utilization level of dedicated machines is lower because of the longer setup requirements of the dedicated machines. Although we do not deal with setup times directly, we still consider this difference between technologies under utilization constraints. Central part of the constraint 13 calculates the required total processing times of each cell and machine type. The constraints 14 and 15 control the total number of machines in a cell. We could have different cell size limitations for the flexible and dedicated cells, if necessary. The constraint 16 controls the loading of a part on a machine. The constraint 17 logically controls the intercellular movement of a part. We also have a set of integrality and nonnegativity constraints as described while defining the decision variables above.

\section{Solution approach}

Since the proposed mathematical model has a large number of binary and integer variables, and nonlinear constraints, it is difficult to obtain an optimal solution to the proposed model in a reasonable computation time. Furthermore, the objectives 1 and 4 have quadratic functions of the decision variables. In order to solve this problem in an acceptable computation time, we propose a local search heuristic as outlined below.

Stage 1. Finding an Initial Solution

Step 1.1 (Data generation) Calculate the $c_{i d}$ and $c_{i f}$ for each part, and the modified Jaccard dissimilarities, $D M J_{i j}$, for each pair of parts.

Step 1.2 (Fuzzy analysis) Perform a fuzzy analysis that uses dissimilarity coefficients and provides a list of membership coefficients for each part.

Step 1.3 Initial part family formation and technology selection

Step 1.4 Initial machine group formation 


\section{Step 1.5 Feasibility Check}

Stage 2. Implement a filtered beam search algorithm to improve the initial solution.

The details of each step are discussed below.

\subsection{Stage 1 -Finding an initial solution}

At this stage of the algorithm, an initial solution is found under consideration of variety costs and operational similarity between parts. Intercellular movements of parts are expected to be at a better level in the initial solution compared to the final solution since the other objectives are not taken into consideration at this first stage.

In Step 1.2, we have adapted the fuzzy algorithm of Kaufmann and Rousseeuw (1990) to find a list of membership coefficients for each part as discussed in the Appendix. We utilize the advantage of the fuzzy clustering over hard clustering. Fuzzy clustering yields much more detailed information on the structure of the data. In the CMSD problem, fuzzy clustering eases the alternative solution generation process at the local search stage. It provides a quantifiable basis where to move the candidate part in the next iteration. We make use of membership matrix frequently in our solution approach as demonstrated in the following numerical example. Although it looks more reasonable to assign each part to the cluster with the maximum membership value, other performance measures such as investment costs, capacity utilizations, etc., might force us to assign this part to some other cluster. This matrix gives us a qualitative measure to compare different clusters.

Example 2. Let the output of a fuzzy analysis performed on the dissimilarity matrix of a CMSD problem is as presented in Table 2, where c1, c2, c3 represent the possible clusters of the system. In this output, we read that it is $50 \%$ beneficial for the part 1 to be in cluster $\mathrm{c} 1$, $20 \%$ beneficial to be in cluster $\mathrm{c} 2$ and $30 \%$ beneficial to be in cluster $\mathrm{c} 3$ in terms of operational similarities. The decision maker has the chance to choose among these alternatives. While choosing cluster $\mathrm{c} 2$ for part 10 is an obvious alternative, for part 2 all clusters are candidates to be assigned in.

After completing the initial calculations, the proposed algorithm identifies the initial part families in Step 1.3. At this step, each part is assigned to the cluster where it has the greatest membership value. The opening of cells are decided at this point in the algorithm. If a cluster

Table 2 An example fuzzy membership matrix

\begin{tabular}{lllllll}
\hline Membership & $\mathrm{c} 1$ & $\mathrm{c} 2$ & $\mathrm{c} 3$ & $c_{i d}$ & & Initial cluster \\
\hline p1 & 0.50 & 0.20 & 0.30 & 20 & $\Rightarrow$ & 1 \\
p2 & 0.33 & 0.32 & 0.35 & 19 & $\Rightarrow$ & 3 \\
p3 & 0.20 & 0.60 & 0.20 & 7 & $\Rightarrow$ & 2 \\
p4 & 0.25 & 0.30 & 0.45 & 5 & $\Rightarrow$ & 3 \\
p5 & 0.25 & 0.25 & 0.50 & 8 & $\Rightarrow$ & 3 \\
p6 & 0.75 & 0.15 & 0.10 & 11 & $\Rightarrow$ & 1 \\
p7 & 0.80 & 0.15 & 0.05 & 4 & $\Rightarrow$ & 1 \\
p8 & 0.15 & 0.70 & 0.15 & 34 & $\Rightarrow$ & 2 \\
p9 & 0.25 & 0.40 & 0.35 & 20 & $\Rightarrow$ & 2 \\
p10 & 0.05 & 0.90 & 0.05 & 37 & $\Rightarrow$ & 2 \\
p11 & 0.30 & 0.55 & 0.15 & 32 & $\Rightarrow$ & 2 \\
\hline
\end{tabular}


is not selected by any of the parts, the corresponding cell is not considered any more in the algorithm.

The technology of the cell is determined based on the market characteristics and the operational similarity between parts. It is determined by the average product variety costs of the associated cluster. If the member parts of a cell tend to have unstable market characteristics, i.e. high variety costs ( $c_{i d}$ 's), it is good to open a cell with flexible technology to process these parts. However, if the general tendency of the member parts is stability in terms of design and demand, i.e. low variety costs $\left(c_{i d}\right.$ 's), the algorithm prefers to open a cell with dedicated technology. Although the flexible cells are more promising in terms of handling changes more effectively than dedicated cells, we should note that there might be costs associated with high number of changes in the future in flexible cells, too. Since we do not consider future periods in our model, we just assume that these costs are lower than the costs involved in a dedicated cell.

However, FMS cells are often only flexible within a certain range of products. If processing requirements change often (high number of design changes) than there are also a lot of costs involved in the FMS cell (writing new CNC programs, new fixtures, tools, etc.).

Example 3. Let the threshold value be 15 and the product variety costs and fuzzy membership matrix values of a problem be as given in Table 2. Each part is assigned to the cluster where it has the greatest membership value. In such a configuration, the algorithm opens all the cells since each cluster has members. To select the technology of each cell, the average variety costs are calculated:

Average Cost of Cluster $1=\frac{20+11+4}{3}=11.66<15$

Average Cost of Cluster $2=\frac{7+34+20+37+32}{5}=26>15$

Average Cost of Cluster $3=\frac{19+5+8}{3}=10.66<15$

As a result, technology of cells 1 and 3 are selected to be the dedicated ones, while cell 2 to be the flexible one.

As an initial configuration of the machines, all the necessary machines are placed in the cell in Step 1.4. Initial operational allocation of parts is also performed simultaneously. If part $i$ in cell $k\left(x_{i k}=1\right)$ requires operation $o\left(P R M_{i o}=1\right)$, and no machine that is capable of the operation exists in the cell, the first machine in the list with appropriate technology and capable of operation $o\left(M C M_{m o}=1\right)$ is assigned to the cell $\left(y_{m k}=1\right)$ and operation is allocated to the machine $\left(z_{\text {imok }}=1\right)$. If there exists a machine $\left(M C M_{m o}=1\right.$ and $\left.y_{m k}=1\right)$, the operation is directly allocated to the machine $\left(z_{\text {imok }}=1\right)$. After allocations, appropriate number of machines are calculated for the cell $k$ according to the upper utilization levels of each machine type as follows:

$$
U t i l_{m k}=\sum_{i, o}\left(z_{i m o k} \cdot \text { Ptime }_{i m o} \cdot D_{i}\right) \quad \text { and } \quad y_{m k}=\left\lceil\frac{U t i l_{m k}}{\operatorname{TCap} \cdot \alpha_{m}}\right\rceil
$$

At this point of the algorithm, initial part families and machine groups are identified. However, this configuration may not be feasible. Since the cells are initially designed to be independent, some machines may exist with very low utilizations in the cells, and sizes of the cells might be larger than the acceptable level. Therefore, we have to check the machine utilization feasibility (the constraint 13) and the cell size feasibility (the constraints 14 and 15). In a cell, if the utilization level of a machine type turns out to be low, first the operations performed on the machine are determined and these operations should be re-allocated. If the 
machine is a dedicated one, parts are forwarded to other cells. We attain feasibility at a cost of number of intercellular movements. If it is a flexible machine, then there is a possibility to find a capable machine within the same flexible cell. If the design is not feasible in terms of cell sizes, algorithm first considers the least utilized machine as a candidate for deletion from the cell. If still the constraint is not satisfied, the algorithm moves a required number of the least utilized machines to another cell.

\subsection{Stage 2 -Local search heuristic}

At the second stage of the algorithm, the monetary and throughput time objectives that have been ignored at the first stage are inserted back in the model. The second stage searches for a better solution in terms of monetary costs, while not deviating much from the other objectives. Filtered beam search technique is the tool employed for the local search, which has three distinguishing parameters beam width, $b$, filter width, $f$, and child width, $c$. The last parameter limits the number of new beams that originate from the same parent beam. Details of the steps are as follows:

\subsection{While not stopping criteria met do}

The algorithm stops either no other candidate machines or parts can be identified in the search space or a pre-specified number of iterations is reached. The details of one search iteration are provided below:

\subsubsection{For each parent solution}

Each iteration begins with generating alternative solutions for each parent solution. Phases of this step are detailed below:

3.3.1.1. Candidate machine selection. If we consider deleting some of the low utilization machines from the cells, we might improve our solution in terms of monetary costs. The best way of finding the low utilization machines is to identify the highest excess capacity machine types in a cell.

$$
\text { Excess }_{m k}=\left(\text { TCap } \cdot \alpha_{m} \cdot y_{m k}\right)-U t i l_{m k}
$$

First filtering mechanism is employed at this step. If the first filter parameter is selected to be $f 1$, first $f 1$ machines are selected for further analysis among the machines with highest excess capacities. Other machines are not considered since the least utilized machines provide the most promising paths around the solution space.

Example 4. Let there exist 5 machines $(m 1, m 2, m 3, m 4, m 5)$ in different cells $(k 1$ dedicated, $k 2$-dedicated, $k 3$-flexible) which have excess capacities. Let $f 1=3$, TCap $=$ 119, 808 min./year and $\alpha_{m}=80 \%$ for dedicated machines and $95 \%$ for flexible machines. Furthermore, let's say

$$
\begin{array}{ll}
\text { Excess }_{m 1, k 1}=75,482 & \text { Excess }_{m 2, k 1}=54,237 \\
\text { Excess }_{m 4, k 2}=80,872 & \text { Excess }_{m 5, k 3}=45,278
\end{array} \quad \text { Excess }_{m 3, k 2}=15,156
$$


The algorithm chooses 3 candidate machines regardless of their technologies; so that $m 4$ of $k 2, m 1$ of $k 1$ and $m 2$ of $k 1$ are selected and new solutions are generated by deleting these machines in the following steps.

3.3.1.2. Candidate part identification. Parts having an operation on the candidate machines are candidates to change clusters. We increase the number of alternatives by considering different part-cluster allocations. Second filtering mechanism is employed at this step. If the second filter parameter is selected to be $f 2$, first $f 2$ part-cluster pairs which have the greatest fuzzy membership coefficients are selected for further evaluation. Highest coefficient parts lead to better solutions since the loss in the objective function values occurs the least with relatively high similarity coefficients.

Example 5. Let the parts, $p 8, p 9$ and $p 11$ of Table 2 have an operation on a candidate machine, and hence they are the candidates to change clusters. Let $f 2=3$, and all the cells be open. Initially, $x_{82}=1, x_{92}=1$ and $x_{11,2}=1$. The algorithm finds the part-cluster pairs that give the greatest fuzzy membership coefficients which are different than their current assignments. Since the filter parameter is equal to 3 , only three of pairs are selected for further evaluation such that $(p 9-k 3),(p 11-k 1)$ and $(p 9-k 1)$ and we have three new configurations $\left[x_{82}^{\prime}=1, \quad x_{93}^{\prime}=1, \quad x_{11,2}^{\prime}=1\right],\left[x_{82}^{\prime \prime}=1, \quad x_{92}^{\prime \prime}=1, \quad x_{11,1}^{\prime \prime}=1\right]$ and $\left[x_{82}^{\prime \prime \prime}=\right.$ $\left.1, \quad x_{91}^{\prime \prime \prime}=1, \quad x_{11,2}^{\prime \prime \prime}=1\right]$.

3.3.1.3. Alternative solution generation. At this point in the algorithm, we generate new alternative solutions based on the candidate machines and parts. At each iteration, for each $b$ parent solutions, the algorithm identifies $f 1$ candidate machines and $f 2$ candidate parts. Then, the number of alternatives (Alt) at each iteration depends on the parameters of the algorithm:

$$
b \cdot f 1 \cdot(f 2+1) \leq A l t \leq b \cdot f 1 \cdot 2 \cdot(f 2+1)
$$

- Without any part transfers, the machine is deleted from the system and parts make intercellular movements for the operation. This design creates a single alternative design.

- Candidate parts are transferred to their candidate cells (one for each alternative) and remaining operations are forwarded to other machines. This design creates $f 2$ alternative designs.

- For each new design, the number of intercellular movements are calculated, and if any part travels more than a pre-defined number of movements, new and revised designs are constructed. This design creates less than $(f 2+1)$ alternative designs.

\subsubsection{Evaluate alternatives}

For each alternative we have 5 different objectives. In order to have a common measure for each criterion, we applied a 0-1 normalization procedure for each objective function at each iteration. At the end of each iteration, objective function values (Equations 1, 2, 3, 4, 5) of each alternative are calculated. Each objective function is normalized compared to the same objective functions of the remaining alternatives of the iteration through following equation:

$$
\operatorname{Normf}_{h}=\frac{f_{h}-\text { GMinf }_{h}}{\operatorname{LMaxf}_{h}-\operatorname{GMinf}_{h}}
$$


We use the minimum value attained at that point in the algorithm as the global minimum value, and the maximum value achieved just in that iteration as the local maximum value. Since our aim is minimization, we should compare the alternatives relative to the best (global minimum) value. On the other hand, we should not lose any information by taking maximum value unnecessarily high. The same equation applies for all of the objectives except the fifth objective, the intercellular movement function. Because of the power of 0 value, which is the global minimum value in general for $f_{5}$, we observed that this function results in very dominant and misleading results. Thus, we change local maximum value to global maximum value for objective 5. The fitness value of each alternative is simply the summation of five normalized objective function values. Best solution is preserved as the incumbent solution, and best $b$ alternatives of the iteration are chosen to be the parents of new iteration. The child mechanism is employed at this step. Child width $c$ limits the number of new parents originating from the same old parent.

Example 6. Let the number of alternatives generated at an iteration is 5 . Let $b$ is 3 , and the objective function values are given in Table 3. Furthermore, the normalized objective function values are given in Table 4. As it is seen from the fitness values of each of the alternatives that Alternative 4 is the best value attained at that point. The incumbent solution, which is represented as the current best, is changed to Alternative 4 at the next iteration. Secondly, the algorithm chooses the parents of the new iteration. They are the best 3 alternatives of this iteration, namely the alternatives 4,1 and 5 .

Table 3 Example 6-Objective function values

\begin{tabular}{lccccl}
\hline Alternatives & $f_{1}$ & $f_{2}$ & $f_{3}$ & $f_{4}$ & $f_{5}$ \\
\hline Initial & 175 & 415 & 315,565 & 978,247 & 0 \\
Current Best & 180 & 457 & 375,672 & 672,169 & 0 \\
Alt 1 & 182 & 415 & 289,614 & 742,245 & 2 \\
Alt 2 & 197 & 567 & 197,723 & 691,837 & 3 \\
Alt 3 & 248 & 502 & 298,521 & 572,893 & 2 \\
Alt 4 & 177 & 499 & 214,345 & 619,361 & 1 \\
Alt 5 & 314 & 467 & 155,983 & 580,347 & 0 \\
G Min & 175 & 402 & 155,983 & 565,741 & 0 \\
Max & 314 & 567 & 375,672 & 978,247 & 8 \\
\hline
\end{tabular}

3.3.3. Go to step 3.3. (Iterative procedure goes on until the stopping criteria is met)

\subsection{Return the final solution}

At the end of the search, the algorithm terminates. Finally, the initial solution, best solution, and global minimum values are reported.

\section{Experimental design}

The proposed algorithm is coded in C language. The code is compiled with Gnu C 5.0 compiler and the set of problems are solved on a $12 \times 400 \mathrm{MHz}$ UltraSparc Station under Solaris 2.7 . 
Table 4 Example 6-Normalized objective function values

\begin{tabular}{lllllll}
\hline & Normf $_{1}$ & Normf $_{2}$ & Normf $_{3}$ & Normf $_{4}$ & Normf $_{5}$ & Fitness \\
\hline Initial & 0.00 & 0.08 & 0.72 & 1.00 & 0.00 & 1.80 \\
Current Best & 0.04 & 0.33 & 1.00 & 0.26 & 0.00 & 1.63 \\
Alt1 & 0.05 & 0.08 & 0.61 & 0.43 & 0.25 & 1.42 \\
Alt2 & 0.16 & 1.00 & 0.19 & 0.31 & 0.38 & 2.04 \\
Alt3 & 0.52 & 0.61 & 0.65 & 0.02 & 0.25 & 2.05 \\
Alt4 & 0.01 & 0.59 & 0.27 & 0.13 & 0.13 & 1.13 \\
Alt5 & 1.00 & 0.39 & 0.00 & 0.04 & 0.00 & 1.43 \\
\hline
\end{tabular}

\subsection{Experimental setting}

Five factors that provide different system properties with their different levels are shown in Table 5. At the production floor, the most important data are the number of parts to be produced and the amount of production volume for each part. Factors A and B together determine the volume data for each part and the amount of production. Table 6 shows the probability of a part to become a high, medium or low volume part. In Table 7, ratios of mean production amount of high and medium volume parts to that of low volume parts are presented.

Table 5 Experimental design factors

\begin{tabular}{|c|c|c|c|c|}
\hline Factors & Definition & Level 0 & Level 1 & Level 2 \\
\hline A & Highest Number of Parts & High Vol. & Low Vol. & Medium Vol. \\
\hline B & $\left(\mathrm{D}_{\text {High Vol.Part }} / \mathrm{D}_{\text {Low Vol. Part }}\right)$ & Low Ratio & High Ratio & - \\
\hline $\mathrm{C}$ & Stability of Environment & Stable & Volatile & - \\
\hline $\mathrm{D}$ & Flexibility & Low & High & - \\
\hline $\mathrm{E}$ & $\left(\right.$ Labor $_{f} /$ Labor $\left._{d}\right)$ & Low Ratio & High Ratio & - \\
\hline
\end{tabular}

Table 6 Factor A

\begin{tabular}{lllll}
\hline Level & High Vol & Med. Vol & Low Vol & Total \\
\hline 0 & 0.35 & 0.50 & 0.15 & 1.00 \\
1 & 0.15 & 0.50 & 0.35 & 1.00 \\
2 & 0.15 & 0.70 & 0.15 & 1.00 \\
\hline
\end{tabular}

Table 7 Factor B

\begin{tabular}{lllc}
\hline Level & High/Low & Med/Low & Low \\
\hline 0 & 8 & 4 & 1 \\
1 & 27 & 9 & 1 \\
\hline
\end{tabular}

In order to receive consistent results, we have to fix the total demand. For each run, the algorithm partitions the total demand to each part type according to the factorial combination. The ratio of the average production amounts to the entire demand comes out to be as it is shown in Table 8. Calculation of the average values are explained below in example 7. After identification of the average values of production amounts, the algorithm assigns volume data for each part in a random fashion $\pm 10 \%$ around the averages. At the end of the demand calculation process, it is verified to maintain the constant demand in total. 
Table 8 Average demand ratios

\begin{tabular}{lllllll}
\hline Setting & 00 & 01 & 10 & 11 & 20 & 21 \\
\hline Ratio for Low Avg. & 0.202 & 0.071 & 0.282 & 0.112 & 0.241 & 0.095 \\
Ratio for Med. Avg. & 0.808 & 0.639 & 1.128 & 1.008 & 0.964 & 0.855 \\
Ratio for High Avg. & 1.616 & 1.917 & 2.256 & 3.024 & 1.928 & 2.565 \\
\hline
\end{tabular}

Table 9 Demand-Age ratios

\begin{tabular}{llllll}
\hline Demand & P1 & P2 & P3 & P4 & P5 \\
\hline High & 0.00 & 0.15 & 0.35 & 0.35 & 0.15 \\
Medium & 0.10 & 0.30 & 0.15 & 0.15 & 0.30 \\
Low & 0.50 & 0.25 & 0.00 & 0.00 & 0.25 \\
\hline
\end{tabular}

Example 7. Take the factorial setting for factors A and B as 0 and 1, respectively, favoring the high volume environment. According to the setting 0 of factor A, 35 of 100 parts have high volume, 50 of 100 have medium and 15 of 100 have low production volume. According to the setting 1 of factor B, high amount means 27 times of low amount, and medium amount equals 9 times of low amount.

$$
\begin{aligned}
\text { Mean Low Volume } & =\frac{\text { Total Demand } \cdot \frac{15 \cdot 1}{35 \cdot 27+50 \cdot 9+15 \cdot 1}}{N \cdot \frac{15}{100}} \\
& =\frac{\text { Total Demand }}{N} \cdot 0.071
\end{aligned}
$$

The numerator of the Eq. (18) gives the concentration of the low volume parts in the entire system. The denominator is the number of low volume parts in the system. On the other hand, (Mean Med Volume) and (Mean High Volume) equal to (Total Demand/ $N$ ) times 0.639 or 1.917 , respectively.

We assume the traditional product life cycle curve as shown in Fig. 1. The associated parameters used in the design are provided in Table 9. In the table, the probability of a part to have a position in each period of the life cycle is presented. In order to be in accordance with the traditional life cycle curve, we assumed that no part might have a high demand in its initial period of lifetime, and no part might have low demand if it is in its third or fourth life periods. For example, if a part has low demand, according to the traditional product life cycle curve, it is in its first period of life with probability 0.50 , in its second life period with probability 0.25 , and in its fifth life period with probability 0.25 . There exist direct relation between age, demand and design patterns of the part. The amount of this relation is affected by the market characteristics. The market might demand design changes either frequently (unstable) or seldom (stable). This triple relation is presented in Table 10. For example, if a part has a high demand, and it is in its second life period, the probability of having a stable design is 0.50 in a stable market, whereas 0.20 in an unstable market.

We understand the machine flexibility as the operational capability of the machines. We assumed that the requirement frequency of an operation shows the expected capability of a machine on that operation. If the level of factor D is 1, more flexibility is favored. Otherwise, machines are designed to be less capable than expected as presented in Table 11. 
Table 10 Factor $\mathrm{C}$

\begin{tabular}{|c|c|c|c|c|c|c|c|}
\hline \multirow[b]{2}{*}{ Volume } & \multirow[b]{2}{*}{$\begin{array}{l}\text { Life cycle } \\
\text { position }\end{array}$} & \multicolumn{3}{|c|}{ Stable environment 0} & \multicolumn{3}{|c|}{ Unstable environment 1} \\
\hline & & $\begin{array}{l}\text { Stable } \\
\text { design }\end{array}$ & $\begin{array}{l}\text { Moderate } \\
\text { design }\end{array}$ & $\begin{array}{l}\text { Volatile } \\
\text { design }\end{array}$ & $\begin{array}{l}\text { Stable } \\
\text { design }\end{array}$ & $\begin{array}{l}\text { Moderate } \\
\text { design }\end{array}$ & $\begin{array}{l}\text { Volatile } \\
\text { design }\end{array}$ \\
\hline High & 2 & 0.50 & 0.30 & 0.20 & 0.20 & 0.30 & 0.50 \\
\hline High & 3 & 0.60 & 0.30 & 0.10 & 0.40 & 0.30 & 0.30 \\
\hline High & 4 & 0.60 & 0.30 & 0.10 & 0.40 & 0.30 & 0.30 \\
\hline High & 5 & 0.50 & 0.30 & 0.20 & 0.20 & 0.30 & 0.50 \\
\hline Medium & 1 & 0.45 & 0.30 & 0.25 & 0.25 & 0.30 & 0.45 \\
\hline Medium & 2 & 0.50 & 0.30 & 0.20 & 0.20 & 0.30 & 0.50 \\
\hline Medium & 3 & 0.55 & 0.30 & 0.15 & 0.35 & 0.30 & 0.35 \\
\hline Medium & 4 & 0.55 & 0.30 & 0.15 & 0.35 & 0.30 & 0.15 \\
\hline Medium & 5 & 0.50 & 0.30 & 0.20 & 0.20 & 0.30 & 0.50 \\
\hline Low & 1 & 0.30 & 0.30 & 0.40 & 0.10 & 0.30 & 0.60 \\
\hline Low & 2 & 0.35 & 0.30 & 0.35 & 0.15 & 0.30 & 0.55 \\
\hline Low & 5 & 0.35 & 0.30 & 0.35 & 0.15 & 0.30 & 0.55 \\
\hline
\end{tabular}

Table 11 Factor D

\begin{tabular}{lc}
\hline Level & Range \\
\hline 0, Less Flexible & $-20 \%$ \\
1, More Flexible & $+20 \%$ \\
\hline
\end{tabular}

A representative example is provided below. After construction of the machine capability matrix with respect to these probabilities, a control mechanism checks the matrix whether a meaningful matrix is produced or not.

Example 8. Take the given part requirement matrix as the input data to the algorithm. According to this part requirement matrix, we calculate the flexibility probability expectation matrix with the following formula:

$$
\text { Expected Probability }=\frac{\text { Number of } 1 \text { 's in the column }}{\text { Total number of parts }}
$$

\begin{tabular}{c|lll} 
PRM & \multicolumn{3}{|c}{ Operations } \\
\hline Parts & 1 & 2 & 3 \\
\hline 1 & 1 & 0 & 1 \\
2 & 1 & 0 & 0 \\
3 & 0 & 1 & 1
\end{tabular}

\begin{tabular}{c|ccc} 
MCM Probability & \multicolumn{3}{|c}{ Operations } \\
\hline Machines & 1 & 2 & 3 \\
\hline fm1 & 0.67 & 0.33 & 0.67 \\
fm2 & 0.67 & 0.33 & 0.67 \\
fm3 & 0.67 & 0.33 & 0.67
\end{tabular}

Each figure in the table shows the probability of the machine to be capable of the operation, i.e. the probability of machine capability matrix (MCM) entry to be 1 . Thus, $M C M_{31}=1$ with the expected probability of 0.67 , and 0 otherwise. Let's assume that the design is constructed in order to favor more flexibility. Let the range parameter be $20 \%$ and our random number generator gives 0.50 . Then, the effective probabilities to produce 1 's in the $M C M_{31}$ increases by factor $(1+(0.20 \cdot 0.50))$. Thus, $M C M_{31}=1$ with the expected probability of $0.74(=0.67 \cdot 1.10)$, and 0 otherwise.

Labor costs get higher when computers are integrated in the system. Not only the operator is paid more with ratio $O R$, but also there exists supplementary labor who is 
Table 12 Factor E

\begin{tabular}{lll}
\hline Level & Operator ratio & Supplementary ratio \\
\hline 0, Low Level & 2 & 0.20 \\
1, High Level & 5 & 0.50 \\
\hline
\end{tabular}

Table 13 Machining center specifications

\begin{tabular}{llllll}
\hline & SQT & SQT & SQT \\
& 200 & $200 \mathrm{M}$ & $\begin{array}{l}\text { Integrex } \\
200 \mathrm{MY}\end{array}$ & $\begin{array}{l}\text { Variaxis } \\
500\end{array}$ \\
\hline Chuck Size & $8^{\prime \prime}$ & $8 ”$ & $8 ”$ & $8 ”$ & - \\
Max.Diameter & $350 \mathrm{~mm}$ & $300 \mathrm{~mm}$ & $300 \mathrm{~mm}$ & $540 \mathrm{~mm}$ & - \\
Spindle speed & $5000 \mathrm{rpm}$ & $5000 \mathrm{rpm}$ & $5000 \mathrm{rpm}$ & $5000 \mathrm{rpm}$ & $12000 \mathrm{rpm}$ \\
RTS speed & - & $4500 \mathrm{rpm}$ & $4500 \mathrm{rpm}$ & $10000 \mathrm{rpm}$ & - \\
Axes & $\mathrm{X}, \mathrm{Z}$ & X,Z,C & X,Z,C,Y & X,Z,C,Y,B & X,Z,C,Y,A \\
Represent & 2 op.s & 3 op.s & 4. op.s & 5 op.s & $\geq 6$ op.s \\
Average & Euro & Euro & Euro & Euro & Euro \\
Investment & 100.000 & 130.000 & 150.000 & 175.000 & 200.000 \\
\hline
\end{tabular}

responsible from the software, maintenance, etc. Supplementary labor is paid with $S R$ ratio to the dedicated machine labor. While one operator is enough for the entire flexible cell, each machine requires an operator in the dedicated cell. The labor cost values are calculated as follows: $\operatorname{Tot}_{f k}=\left(\right.$ Labor $\left._{d} \cdot O R\right)+\sum_{m}\left(\right.$ Labor $\left._{d} \cdot S R \cdot y_{m k}\right) \quad \forall$ flexible cell $k$, and $\operatorname{Tot}_{d k}=\sum_{m}\left(\right.$ Labor $\left._{d} \cdot y_{m k}\right) \quad \forall$ dedicated cell $k$.

There should exist virtual part groups in order to apply cellular manufacturing. Random data should not be used. We have employed the part-machine incidence matrices of Chandrasekharan and Rajagopalan (1989). Authors have provided many data sets ranging in different structures. We have implemented the data set 6 in our experimental design since it is neither perfectly groupable nor badly structured (grouping efficiency is $73.43 \%$ ). This machine-part incidence matrix is utilized as the part requirement matrix of our algorithm. Derived from this data, number of parts in the system is 40 , number of operations is 24 , and number of dedicated machines is also 24 . The number of available flexible machines is assumed to be 12 .

Machine investment cost is another key concept in the proposed algorithm. The data is adapted from Mazak Corporation. We analyzed the properties of numerous types of flexible machines produced by Mazak, and selected some benchmark machine types that are the most representative ones to be employed in our study. Machining center properties of the selected types are provided in Table 13 . We have selected the machines with nearly same size specifications while with different operational capabilities. The prices are disguised without altering the real ratios.

We employed universal turning machine as a basis for the dedicated technology with its worth of Euro 15.000 on the average. The number of axes in a machining center significantly affects the operational capability of these machines. SQT 200 is one of the least capable flexible machines. SQT 200M and SQT 200MY are multi-tasking CNC turning centers that can turn and mill a workpiece in a single machine setup. Integrex Series machines are the most widely used multi-tasking machine tools in the world. Integrex $200 \mathrm{Y}$ is a representative machine type for our 5-operations case with its 5 axes $(\mathrm{X}, \mathrm{Z}, \mathrm{C}, \mathrm{Y}, \mathrm{B})$ available for different 
operations. Variaxis Series machines are multi-face and simultaneously controlled 5-axis double-column machining centers. We have selected Variaxis 500 to represent the flexible machines that are the most capable ones in terms of operations. Under consideration of lifetimes of the machines (10 years for dedicated technology, 15 years for flexible technology), annual machine investment costs are selected randomly in a range of $\pm 5 \%$ of investment costs. Maintenance costs of machines are simply taken to be $10 \%$ of investment costs. Number of machines in each cell has an initial upper bound (UBK) of 15 machines. This initial limit tends to be halved at the end of the search procedure. In the final design, total annual demand $\left(\sum_{i} D_{i}\right)$ is taken to be 50,000 units and number of cells is assumed to be 4 . Theoretical capacity of machines is calculated as follows:

\section{TCap $=48$ min $/$ hour $\cdot 8$ hours $/$ day $\cdot 6$ days $/$ week $\cdot 52$ weeks $/$ year $=119,808$ min $/$ year}

Because of setups and maintenance stops, the theoretical capacities can never be achieved. Upper level of utilization $\left(\alpha_{m}\right)$ for dedicated machines is taken to be $80 \%$, and for flexible machines it is $95 \%$. The lower utilization bound $\left(\beta_{m}\right)$ is determined to be the same for both types of machines as $5 \%$. The processing times of parts on dedicated machines are selected randomly from the uniform interval $U[5,10]$ whereas that of flexible machines are selected from the uniform interval $U[5,15]$. Load-unload times of parts on dedicated machines are selected randomly from the uniform interval $U[1,2]$ whereas that of flexible machines are selected from the uniform interval $U[1.5,2.5]$.

\subsection{Experimental results}

The experimentation is performed by a $\left(3 \cdot 2^{4}\right)$ full factorial design with the factors detailed in the previous section. With 5 different random number seeds, 240 randomly generated problems are solved by the proposed algorithm and a challenger algorithm with different beam widths ( $b=3$ and $b=6$ ). As far as we know, there does not exist hybrid technology approaches in the literature handling product variety, and most of the existing cellular manufacturing literature assumes dedicated technology. Therefore, we consider a fully dedicated technology as the challenging algorithm in our case. For the challenger, we have re-constructed the proposed algorithm such that the available technology is the dedicated technology for the whole system. We utilized the summation of normalized objective function values as the performance measures. The relative difference between the two challenger algorithms shows the performance of the algorithms for different factorial combinations.

$$
\text { Performance Measure }=\frac{\text { Dedicated }- \text { Hybrid }}{\text { Hybrid }} * 100
$$

Performance measure computes the difference between results of the proposed hybrid algorithm and challenger dedicated algorithm. In the measure, we rate this difference compared to the value of the proposed algorithm. The overall improvement that the hybrid algorithm achieves is $53.6 \%$. Table 14 presents the average values for each factor level. The improvement percentages for each factor shown are the averages of all possible combinations of levels of other factors. They represent the main effects considered in the study.

As expected, when the general tendency is low volume (Factor A is at level 1), hybrid technologies prove much better. Factor B, on its own, does not provide a significant effect on the system. Factor $\mathrm{C}$ is the most effective factor in the entire system. The stability of the environment is a crucial attribute which should never be discarded. It is seen from the 
Table 14 Improvement percentages for each factor

\begin{tabular}{llllll}
\hline & \multicolumn{2}{c}{ Factor } & & \multicolumn{3}{c}{ Performance measure } \\
\cline { 5 - 6 } Type & Level & & Min & Avg & Max \\
\hline A & 0 & & -39.3 & 45.4 & 165.9 \\
A & 1 & & -29.8 & 68.7 & 146.7 \\
A & 2 & & -39.2 & 46.7 & 160.9 \\
B & 0 & -39.3 & 54.4 & 152.8 \\
B & 1 & -39.2 & 52.8 & 165.9 \\
C & 0 & -39.3 & 27.9 & 145.6 \\
C & 1 & -29.1 & 79.3 & 165.9 \\
D & 0 & -39.2 & 45.5 & 165.9 \\
D & 1 & -39.3 & 61.7 & 152.8 \\
E & 0 & -36.3 & 60.2 & 165.9 \\
E & 1 & -39.3 & 47.1 & 120.6 \\
\hline
\end{tabular}

results that when the market gets more volatile, the need for hybrid manufacturing systems significantly rises. Under high product variety assumption, the improvement we get by implementing hybrid strategies instead of dedicated technology is as high as $80 \%$ on the average. When the machines get more capable in terms of operations (Factor D is at level 1), it is easier to justify the high investment of these machines. When the difference between flexible and dedicated labor costs decreases (Factor $\mathrm{E}$ is at level 0), in other words, to hire a flexible machine operator becomes cheaper, the algorithm tends to implement hybrid technology systems, as expected.

Since we use the summation of normalized objective function values as the performance measures, all objectives have the equal weight on the final performance values. Regarding the objectives, it should be noted that Factors A, B, and C are related to the variety costs objectives 1 and 2, Factor $\mathrm{D}$ is related to machine costs and intercellular movement objectives 3, 4 and 5, and Factor $\mathrm{E}$ is related to machine costs objective 4. We expect Factor $\mathrm{C}$ to have a greater effect on the results than Factors A and B because of the definition of variety cost function. The design stability was given the highest weight during the calculation of variety costs. However, it is surprising for Factor $\mathrm{C}$ to have a greater effect than monetary and technological factors. This result shows that it is crucial to implement hybrid technologies in unstable environments regardless of the high investment costs.

The results for each objective are shown in Table 15. It is observed that the similarity achieved in hybrid approach is better. The study shows the necessity of integration of operational flexibility of machines in the similarity context. Further, although we expect that the search algorithm worsens the similarity objective, we have found a better solution after the search stage. This shows that it is better to perform search with the help of fuzzy membership coefficients in cellular manufacturing system design problems. We have the same product variety results in three phases of the dedicated technology, since all the parts are always in dedicated cells. It is observed that the product variety costs are significantly higher when we force dedicated technology. The study shows the necessity of hybrid technology implementation at the production floor to hedge against the variations in the market. Throughput time objective is used as a secondary objective in the search algorithm. It affects the results indirectly at the evaluation step, together with the other objectives. The other noteworthy objective of this study is the machine investment, maintenance and labor costs objective $\left(f_{4}\right)$. Costs of hybrid approach are significantly higher, because of the flexible machines integrated 
Table 15 Objective function values

\begin{tabular}{|c|c|c|c|c|c|c|c|c|c|c|}
\hline & \multicolumn{3}{|c|}{ Initial } & \multicolumn{3}{|c|}{ Beam Width $=3$} & \multicolumn{3}{|c|}{ Beam Width $=6$} \\
\hline & & Min & Avg & Max & Min & Avg & Max & Min & Avg & Max \\
\hline \multirow[t]{2}{*}{$f_{1}$} & Ded. & 518.3 & 518.3 & 518.3 & 436.4 & 448.7 & 478.3 & 435.0 & 447.0 & 518.3 \\
\hline & Hyb. & 6.8 & 161.2 & 491.2 & 1.5 & 123.0 & 443.4 & 1.5 & 121.0 & 444.6 \\
\hline \multirow[t]{2}{*}{$f_{2}$} & Ded. & 414.0 & 707.9 & 995.0 & 414.0 & 707.9 & 995.0 & 414.0 & 707.9 & 995.0 \\
\hline & Hyb. & 414.0 & 600.1 & 839.0 & 372.0 & 587.6 & 839.0 & 370.0 & 588.0 & 839.0 \\
\hline \multirow[t]{2}{*}{$f_{3}$} & Ded. & 160,902 & 219,163 & 266,576 & 399,027 & 470,915 & 528,848 & 190,964 & 466,468 & 528,848 \\
\hline & Hyb. & 225,233 & 500,127 & 751,864 & 345,757 & 545,828 & 663,791 & 471,284 & 543,840 & 663,791 \\
\hline \multirow[t]{2}{*}{$f_{4}$} & Ded. & 326,715 & 492,928 & 653,021 & 221,557 & 267,569 & 291,480 & 221,557 & 268,737 & 326,715 \\
\hline & Hyb. & 450,230 & 681,907 & 976,300 & 279,163 & 473,421 & 734,839 & 279,163 & 468,692 & 680,833 \\
\hline \multirow[t]{2}{*}{$f_{5}$} & Ded. & 3 & 10.8 & 22 & 30 & 39.7 & 47 & 14 & 39.7 & 49 \\
\hline & Hyb. & 0 & 2.77 & 20 & 0 & 16.9 & 53 & 0 & 17.2 & 47 \\
\hline
\end{tabular}

in the system. When the overall averages are analyzed, we observe that the justification of high investment of flexible systems is achieved easily. The last objective is the intercellular movement objective $\left(f_{5}\right)$. Although the initial solution is constructed to form independent cells, feasibility forces the parts make intercellular movements at the last step. The number of intercellular movement of parts in hybrid approach is less than the dedicated challenger. Integration of flexible systems decreases the exceptional parts in the system, increasing the efficiency of the CMSD.

In Table 16, improvements of hybrid approach against the dedicated approach for several selected factorial combinations are presented. Setting (101-) constructs a system in an unstable marketing environment which has more number of low volume parts and with relatively high average demand ratio. Hybrid technology selection for cellular manufacturing systems prove more than $100 \%$ better than the fully dedicated cellular manufacturing systems in such an environment. The study shows that the hybrid technology approach to CMSD problem works much better than the dedicated technology in unstable markets with high product varieties. Further, if the flexible labor cost is low, i.e. in the setting (101-0), average improvement reaches up to $116 \%$. In setting $(-110)$, algorithm constructs a system in an unstable environment which has highly capable machines and low costs of flexible labor. Improvement provided by the proposed algorithm is more than $95 \%$ regardless of the volumes of the parts. Even in setting (01110), where (01-) provides a high volume dominant production environment, the average improvement is still around $95 \%$. Moreover, when the environment is set to favor low volume parts (1-110), average improvement achieved becomes $108 \%$. There are cases where the investment in flexible technology cannot be justified. When the system provides high demand ratio for high volume parts in stable environments, or less capability of

Table 16 Improvement percentages for selected factor combinations

\begin{tabular}{lccc}
\hline \multirow{2}{*}{$\begin{array}{l}\text { Factors } \\
\text { (ABCDE) }\end{array}$} & Min & Avg & Max \\
\cline { 2 - 4 }$(101--)$ & 55.1 & 103.8 & 146.7 \\
$(101-0)$ & 64.1 & 116.1 & 146.7 \\
$(-110)$ & 49.7 & 98.9 & 152.8 \\
$(1-110)$ & 81.3 & 107.9 & 137.9 \\
\hline
\end{tabular}


Table 17 CPU times in seconds

\begin{tabular}{|c|c|c|c|c|c|c|c|c|c|}
\hline \multirow[b]{2}{*}{ Algorithm } & \multicolumn{3}{|c|}{ Initial } & \multicolumn{3}{|c|}{ Beam width $=3$} & \multicolumn{3}{|c|}{ Beam width $=6$} \\
\hline & Min & Avg & Max & Min & Avg & Max & Min & Avg & Max \\
\hline Dedicated & 5.8 & 6.3 & 6.9 & 45.6 & 104.1 & 169.1 & 72.5 & 199.2 & 330.2 \\
\hline Hybrid & 5.7 & 6.3 & 7.5 & 9.0 & 93.6 & 325.5 & 14.9 & 189.0 & 619.5 \\
\hline
\end{tabular}

the flexible machines and higher cost of flexible labor, the improvements decrease to negative values favoring the dedicated technology. Dedicated systems are still very powerful when the market is stable and production volumes are high. Negative performance values show that the proposed algorithm favors flexible technologies even when dedicated technology would be enough.

Another significant attribute of the algorithm is the beam width selection. As the beam width gets larger, the solution space that is inspected also enlarges. This brings a higher cost of computation time. Table 17 shows the final average CPU times of the algorithms. Both algorithms provide $20 \%$ improvement from the initial solution at the search phase. This improvement is worth computation. However, the quality of the final solution of larger beam width is only in $1 \%$ neighborhood of the smaller beam width. We believe this much improvement is not worth twice much computation time.

\section{Conclusion}

In today's world, while the customers are expecting product variety, it makes the manufacturing processes considerably difficult. This has implications for integration of flexible manufacturing systems. Group technology applications has further benefits at the production floor. We propose a model that makes technology selection and cell formation to hedge against the changing market dynamics. In our multi-objective study, we modified a well known similarity measure to handle the operational capability of available technology. In order to integrate the market characteristics in our model, we proposed a new cost function. This is the first study to assign costs for design instabilities and demand variations for the CMSD problem. The study shows the significance of hybrid technology implementation at the production floor to cope with the variations in the market. We also show the necessity of integration of operational capability of machines in the similarity context. Integration of flexible systems decreases the exceptional parts in the system, while increasing the efficiency of the CMSD. Some future research directions can be summarized as follows. We emphasize the design problem in this study, although the impact of operational issues, such as cell loading and scheduling, can be analyzed. The proposed model does not take into account neither the operation sequences of parts nor the layout of the facility. Integration of these attributes in the model can be a challenging study.

\section{Appendix: Fuzzy analysis algorithm}

Fuzzy clustering is a generalization of partitioning. In a partition, each object of the data set is assigned to one and only one cluster. Therefore, partitioning methods produce a hard clustering, because they make a clear-cut decision for each object. On the other hand, a fuzzy 
clustering method allows for some ambiguity in the data. We use the following notation for the fuzzy analysis algorithm of Kaufmann and Rousseeuw (1990):

$C$ : minimization objective function

$k \quad$ : maximum number of clusters

$n \quad:$ number of parts

$u_{i v}:$ fuzzy membership coefficient of part $i$ in cluster $v$

$d_{i, j}$ : given dissimilarities between parts $i$ and $j$

$$
\min C=\sum_{v=1}^{k} \frac{\sum_{i, j=1}^{n}\left(u_{i v}\right)^{2} \cdot\left(u_{j v}\right)^{2} \cdot d_{i, j}}{2 \cdot \sum_{j=1}^{n}\left(v_{j v}\right)^{2}}
$$

The algorithm attempts to minimize the objective function $C$ in Eq. (19). It contains the dissimilarities $\left(d_{i, j}\right)$ and the membership coefficients that we are trying to find. The sum in the numerator ranges over all pairs of parts $(i, j)$. Since $(j, i)$ also occurs, the sum is divided by 2. The outer sum is over all clusters, so the objective is comprehensive. The algorithm works iteratively and stops when the objective function converges. The membership functions are subject to the constraints 20 and 21 expressing that memberships cannot be negative and that each part has different membership values distributed over the different clusters which sum up to 1:

$$
\begin{aligned}
& u_{i v} \geq 0 \quad \text { for } i=1, \ldots, n ; v=1, \ldots, k \\
& \sum_{v} u_{i v}=1 \quad \text { for } i=1, \ldots, n
\end{aligned}
$$

Via use of Lagrange multipliers, the corresponding Kuhn and Tucker conditions are calculated taking into account the objective function (19) and the constraints (20,21). The interested reader on the mathematical proof of the algorithm is referred to Kaufmann and Rousseeuw (1990). Having arbitrary initial values for all $u_{i v}$, the algorithm computes new and better membership coefficients at each iteration, until the objective function converges. The proposed iterative algorithm has the following form: (Note that superscripts stand for the number of iteration step)

1 Initialize the membership functions as $u_{i v}^{0}$ for all $i=1, \ldots, n$ and all $v=1, \ldots, k$, taking into account constraints 20 and 21. Calculate the objective function $C^{0}$ by 19 .

In our algorithm, we have $k=4$, and the following arbitrary initial membership coefficients are provided as initial feasible values in terms of the constraints:

$u_{i 1}^{0}=0.10, u_{i 2}^{0}=0.20, u_{i 3}^{0}=0.30$ and $u_{i 4}^{0}=0.40$.

2 Compute for each $i=1, \ldots, n$ the following quantities:

2.1 Compute for each $v=1, \ldots, k$ :

$$
\begin{aligned}
& a_{i v}^{m}=\frac{N u m 1-(N u m 2+N u m 3+N u m 4+N u m 5)}{\operatorname{Den}} \\
& \text { Num } 1=2 \cdot\left(\sum_{j=1}^{i-1}\left(u_{j v}^{m+1}\right)^{2} \cdot d_{i j}+\sum_{j=i}^{n}\left(u_{j v}^{m}\right)^{2} \cdot d_{i j}\right)
\end{aligned}
$$




$$
\begin{array}{r}
\text { Num } 2=\sum_{j=1}^{i-1} \sum_{h=1}^{i-1}\left(u_{j v}^{m+1}\right)^{2} \cdot\left(u_{h v}^{m+1}\right)^{2} \cdot d_{i j} \\
\text { Num } 3=\sum_{j=1}^{i-1} \sum_{h=i}^{n}\left(u_{j v}^{m+1}\right)^{2} \cdot\left(u_{h v}^{m}\right)^{2} \cdot d_{i j} \\
\text { Num } 4=\sum_{j=i}^{n} \sum_{h=1}^{i-1}\left(u_{j v}^{m}\right)^{2} \cdot\left(u_{h v}^{m+1}\right)^{2} \cdot d_{i j} \\
\text { Num } 5=\sum_{j=i}^{n} \sum_{h=i}^{n}\left(u_{j v}^{m}\right)^{2} \cdot\left(u_{h v}^{m}\right)^{2} \cdot d_{i j} \\
\text { Den }=\sum_{j=1}^{i-1}\left(u_{j v}^{m+1}\right)^{2}+\sum_{j=i}^{n}\left(u_{j v}^{m}\right)^{2}
\end{array}
$$

2.2 Compute for each $v=1, \ldots, k$ :

$$
A_{v}=\frac{1 / a_{i v}^{m}}{\sum_{w}\left(1 / a_{i w}^{m}\right)}
$$

2.2.1 if $A_{v} \leq 0 \Rightarrow V 1=V 1 \cup\{u\}$

2.2.2 if $A_{v}>0 \Rightarrow V 2=V 2 \cup\{u\}$

2.3 Put for all $v \in V 1$

$$
u_{i v}^{m+1}=0
$$

2.4 Compute for all $v \in V 2$

$$
u_{i v}^{m+1}=\frac{1 / a_{i v}^{m}}{\sum_{w \in V 2}\left(1 / a_{i w}^{m}\right)}
$$

2.5 Put $V 1=V 2=\emptyset$ and restart from Step 2.1 with the next $i$.

3 Calculate the new objective function value $C^{m+1}$ by Equation (19). If $\left(C^{m} / C^{m+1}-1\right)<$ $\in$, then go to Step 2; otherwise stop.

\section{References}

Abdi MR, Labib AW (2004) Grouping and selecting products: The design key of reconfigurable manufacturing systems. International Journal of Production Research 42(3):521-546

Akturk MS, Balkose O (1996) Part-machine grouping using a multi-objective cluster analysis. International Journal of Production Research 34(8):2299-2315

Bokhorst JAC, Slomp J, Suresh NC (2002) An integrated model for part-operation allocation and investments in CNC technology. International Journal of Production Economics 75(3):267-285

Chandrasekaran MP, Rajagopalan R (1989) Groupability: An analysis of the properties of binary data matrices for group technology. International Journal of Production Research 27(6):1035-1052

Garbie IH, Parsaei HR, Leep HR (2005) Introducing new parts into existing cellular manufacturing systems based on a novel similarity coefficient. International Journal of Production Research 43(5):1007-1037 
Gupta YP, Goyal S (1989) Flexibility of manufacturing systems: Concepts and measurements. European Journal of Operational Research 43:119-135

Kaufmann L, Rousseeuw P (1990) Finding groups in data: An introduction to cluster analysis. John Wiley \& Sons, Inc., New York, NY

Li L, Tirupati D (1994) Dynamic capacity expansion problem with multiple products: Technology selection and timing of capacity additions. Operations Research 42(5):958-976

Rajagopalan S (1993) Flexible versus dedicated technology: A capacity expansion model. The International Journal of Flexible Manufacturing Systems 5:129-142

Ramdas K (2003) Managing Product Variety: An integrative review and research directions. Production and Operations Management 12(1):79-101

Selim HM, Askin RG, Vakharia AJ (1998) Cell formation in group technology: Review, evaluation and directions for future research. Computers and Industrial Engineering 34(1):3-20

Singhal K, Fine CH, Meredith JR, Suri R (1987) Research and models for automated manufacturing. Interfaces 17(6):5-14

Suresh NC, Slomp J (2001) A multi-objective procedure for labor assignments and grouping in capacitated cell formation problems. International Journal of Production Research 39(18):4103-4131

Venkatesan R (1990) Cummins engine flexes its factory. Harvard Business Review 68(2):120-127

Verter V, Dasci A (2002) The plant location and flexible technology acquisition problem. European Journal of Operational Research 136:366-382

Wicks EM, Reasor RJ (1999) Designing cellular manufacturing systems with dynamic part populations. IIE Transactions 31(1):11-20 